\title{
Heart-Lung Machine: Seven Decades of Challenging Work
}

\author{
Yasser Ali Kamal* \\ Department of Cardiothoracic Surgery, Minia University Hospital, El-Minya, Egypt
}

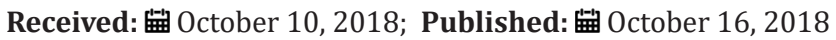

*Corresponding author: Yasser Ali Kamal, Department of Cardiothoracic Surgery, Minia University Hospital, El-Minya, 61519, Egypt

\section{Opinion}

Heart-lung machine (HLM), also called oxygenator pump or cardiopulmonary bypass (CPB) pump (Figure 1), aims to provide extracorporeal circulation (ECC) with maintenance of oxygenated blood flow to the body organs when the device is connected to the arteriovenous system. The HLM is an essential component of openheart surgery to bypass the circulatory system of the heart and lungs, termed CPB. The main principle is to draw the blood from the major veins (venae cavae) to an oxygenator in the HLM where it is re-oxygenated, and then pumped into the arterial system (ascending aorta). Additionally, the myocardial contractility during
CPB can be halted by running a cardioplegic solution containing potassium citrate. The initial concept to constitute an artificial circulation and hence CPB was stated in the 19th century on the basis that the contractility of muscle could be restored by perfusion of an oxygenated blood under pressure to enable better perfusion of isolated organs. Therefore, there was a need to develop an artificial heart-lung system to perfuse an oxygenated solution without interruption of blood flow, which became a real in the beginning of the $20^{\text {th }}$ century after discovery of the ABO blood group system to reduce inconveniences of incompatibility, in addition to discovery of heparin to inhibit coagulation [1].

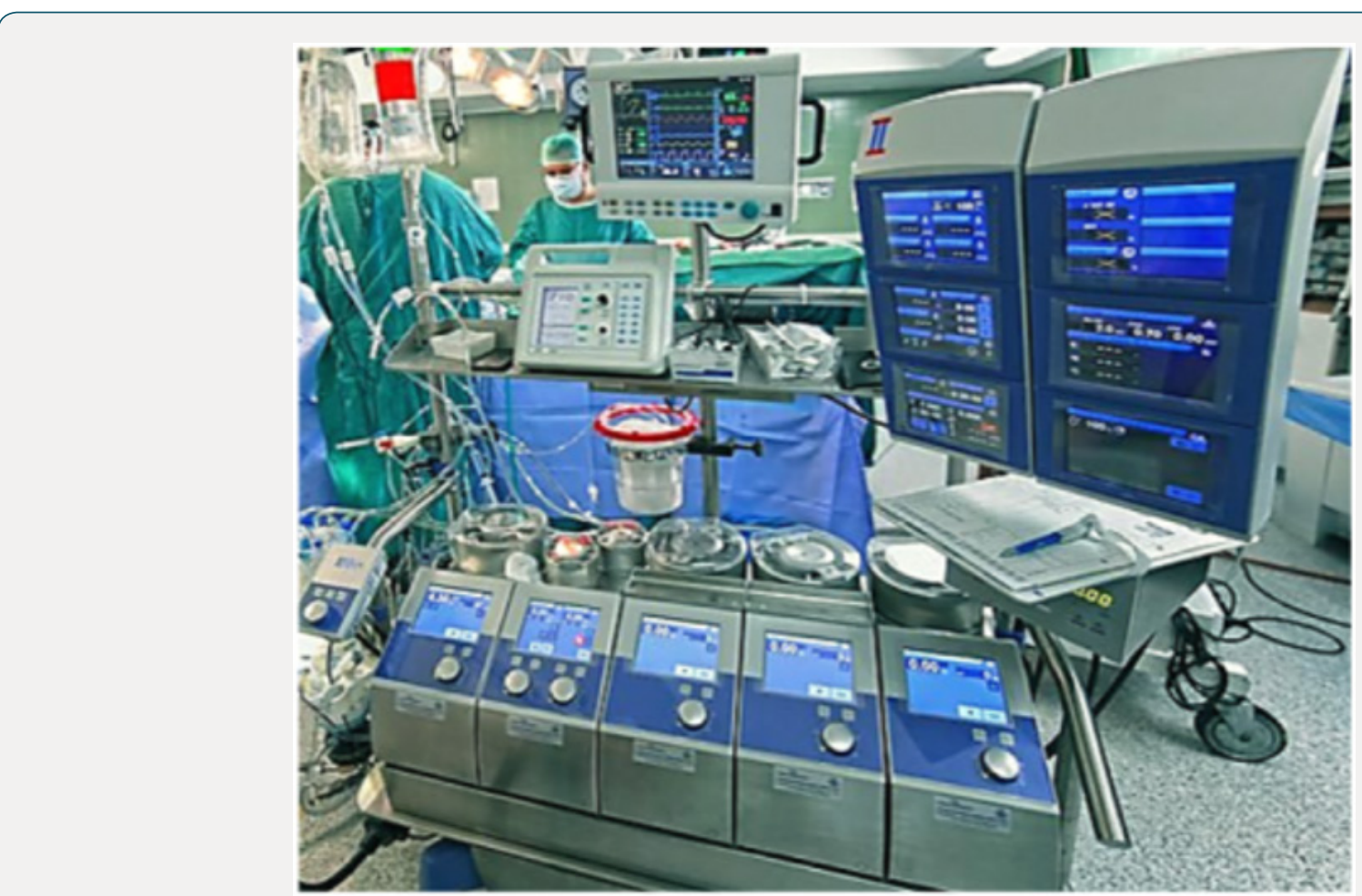

Figure 1: Heart-lung machine in the cardiac operating room: Fifth generation of roller pumps (Sorin Group, Munich) with control and monitoring panels. 
Nowadays, no one can ignore the great clinical role of heartlung machine (HLM) in the development and progress of cardiac surgery, since the first successful clinical use of CPB by John Gibbon Jr on 1953. The practical routine uses of CPB for open heart surgery began in 1955, thanking to the efforts of John Kirklin and Walton Lillehei [2]. Thereafter, many technical and practical advances occurred in HLM and perfusion science in line with advancements in anesthesia and cardiac surgery, to reach about seven decades after its first clinical application with contemporary important developments and promising future. Since its incorporation for cardiac surgery, subsequent important developments have been driven to HLM and CPB. The improvements allowed an increase in the operative time, better myocardial protection, and extension of open-heart surgery to include patients at extreme age or those who have inoperable conditions [3]. The oxygenators of HLM have passed by many transitions from disposable biologic oxygenators in 1960s, to microporous polypropylene oxygenators in 1970s, hollow-fiber oxygenators in 1980s and 1990s, nonporous true diffusion membrane in 2000 s, and more recently screen microfilters to eliminate the need for a separate arterial line. The initial Sigmamotor finger pumps arterial pumps were replaced by roller pumps, centrifugal pumps, and pulsatile mode pumps. In addition, the technical advances in HLM and CPB include improvements in arterial cannulation, venous drainage and cannulation, temperature management, anticoagulation therapy, hemofiltartion, organ preservation, brain protection, and monitoring [4].

The developments in the ECC technology, particularly the use of centrifugal pumps and nonporous true diffusion membrane, lead to development of partial CPB termed extracorporeal membrane oxygenator (ECMO) to prolong life-support systems for patients with heart and/or lung failure [5]. Currently, ECMO is used as a technique to treat critically ill patients with cardiogenic shock and cardiac arrest, to obtain worldwide use of ECMO further improvements in circuit design, oxygenator technology, and portability are required [6]. Despite seven decades of continuous improvements in ECC and HLM, the device was not without its faults because of the possible complications related to inflammatory response, hemodilution, coagulation abnormalities, cerebral dysfunction, and endothelial damage. Multiple strategies have been introduced to reduce the severity of the associated systemic inflammation and organ dysfunction, including anti-inflammatory drugs, novel pharmacologic components of the $\mathrm{CPB}$, and modified surgical techniques, but the efficacy of these strategies is controversial $[7,8]$. To avoid the unfavorable consequences during coronary artery bypass grafting (CABG), exclusion of CPB namely off-pump coronary artery bypass (OPCAB) have been advocated and widely used since its introduction on 1990s [3,9].
Despite the theoretical benefits of OPCAB in reduction of myocardial injury, stroke, neurocognitive impairment, and cardiac mortality, comparisons of OPCAB with on-pump CABG showed favorable short-term and doubtful long-term outcomes. In comparison to on-pump CABG, the benefits of OPCAB regarding reduced need for blood transfusion and reduced postoperative hospital stay may be outweighed by the risk of incomplete ineffective revascularization, with current decline in the worldwide rates of OPCAB [9]. A debate remains regarding the use of HLM as it is an essential element of cardiac surgery, but its use is still faced by some limitations which may constitute more challenges for future investigations. In other words, CPB has a rapid evolution since the clinical introduction of HLM until the current remarkable safety but its perfection is still pending.

In conclusion, invent of HLC with development of CPB constitute the real breakthrough for cardiac surgery. Through approximately seven decades, the clinical evolution of HLM and CPB with a reduction of its early complications encouraged the performance of more cardiac procedures and the involvement of more population in open heart surgery including those with comorbidities and advanced age. However, the complications of CPB remain possible in relation to the associated hemolysis and inflammatory response which indicates more future work to eliminate any intricacy.

\section{References}

1. Passaroni AC, Silva MA, Yoshida WB (2015) Cardiopulmonary bypass: development of John Gibbon's heart-lung machine. Rev Bras Cir Cardiovasc 30(2): 235-245.

2. Hessel II EA (2014) A brief history of cardiopulmonary bypass. Seminars in Cardiothoracic and Vascular Anesthesia 18(2): 87-100.

3. Kumar AS (2001) Heart lung machine; Armament or ornament. Indian J Thorac Cardiovasc Surg 17: 223-224.

4. Hessel II EA (2015) History of cardiopulmonary bypass (CPB). Best Practice \& Research Clinical Anaesthesiology 29: 99-111.

5. Ramakrishna H (2015) Extracorporeal circulation-from cardiopulmonary bypass to extracorporeal membrane oxygenation and mechanical cardiac assist device therapy: A constant evolution. Ann Card Anaesth 18(2): 133-137.

6. Baran DA (2017) Extracorporeal membrane oxygenation (ECMO) and the critical cardiac patient. Curr Transplant Rep 24(3): 218-225.

7. Asimakopoulos G (2001) Systemic inflammation and cardiac surgery: an update. Perfusion 16(5): 353-360.

8. Landis C (2007) Pharmacologic strategies for combating the inflammatory response. The Journal of extra-corporeal technology 39(4): 291-295.

9. Shaefi S, Mittel A, Loberman D, Ramakrishna H (2018) Off-pump versus on-pump coronary artery bypass grafting-A systematic review and analysis of clinical outcomes. J Cardiothorac Vasc Anesth. 
(c) $\left(\begin{array}{l}\text { TY } \\ \text { Commons Attribution } 4.0 \text { License }\end{array}\right.$

To Submit Your Article Click Here: $\quad$ Submit Article

DOI: $10.32474 /$ LOJMS.2018.02.000131

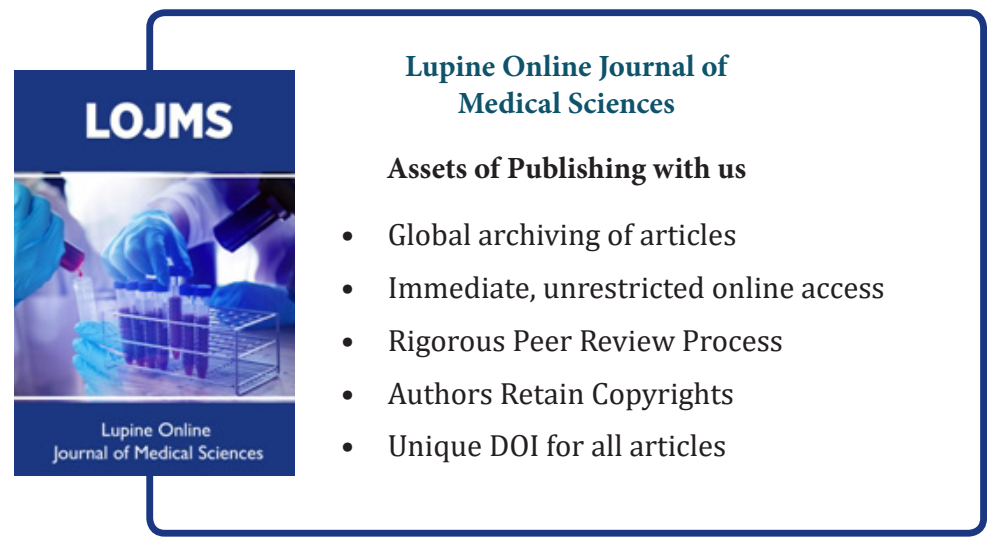

